\title{
The exit times for the diffusion risk model with constant interest
}

\author{
Jingmin $\mathrm{He}^{1, \text { a }}$, Wei Zhang ${ }^{2, b}$ \\ ${ }^{1}$ School of Science, Tianjin University of Technology, Tianjin 300384, P.R. China \\ ${ }^{2}$ School of Mathematics and statistics, Central South University, Changsha, Hunan, 410083, China \\ ankjmhe_2002@163.com, bzhangyunfei78@csu.edu.cn
}

Keywords: Exit time; Confluent hypergeometric function; Strong Markov property; Dynkin's formula; Martingale.

Abstract. This paper investigates the diffusion risk model with constant interest. The Laplace-Stieltjes transforms (LST) of some exit times of the risk process are obtained.

\section{Introduction}

The diffusion risk model with constant interest is described by

$$
U(t)=u+\int_{0}^{t} C(U(s)) d s+\sigma B(t)
$$

where $u$ denotes the initial capital, $C(x)=c+r x, c>0$ represents the premiums income pre unit time and $r>0$ is the constant force of interest. $\{B(t), t \geq 0\}$ is a standard Brownian motion and $\sigma>0$ is the diffusion coefficient. The model (1) is a diffusion risk model with interest which represents that the company can earn investment income at a constant force of interest $r$ when the surplus is positive. When the surplus turns negative, the company is allowed to borrow money at the same force of interest $r$.

For any interval $[b, a]$, where $b<u<a$, define the first hitting time of the upper barrier $a$ for the risk process $\{U(t), t \geq 0\}$ to be

$$
T_{a}=\left\{\begin{array}{l}
\inf \{t \geq 0, U(t)=a\}, \\
\infty, \quad \text { if } U(t) \neq a \text { for all } t \geq 0 .
\end{array}\right.
$$

Correspondingly, define the first hitting time of the lower barrier $b$ for the risk process $\{U(t), t \geq 0\}$ to be

$$
T_{b}=\left\{\begin{array}{l}
\inf \{t \geq 0, U(t)=b\}, \\
\infty, \quad \text { if } U(t) \neq b \text { for all } t \geq 0 .
\end{array}\right.
$$

Then $T_{a, b}=T_{a} \wedge T_{b}$ is the first exit time of the process $\{U(t), t \geq 0\}$ from the interval $(b, a)$. This paper investigates the Laplace-Stieltjes transforms (LST) of the exit times. The similar subject of this paper is considered by some authors. Alili, Patie and Pedersen[1] mainly considered the first hitting time of an Ornstein-Uhlenbeck process. Chiu and Yin[2-4] investigated some passage times of the reserve-dependent risk process and the spectrally negative Lévy process. dos Reis[5] and Gerber[6] mainly studied some stopping times of the classical risk process. Jacobsen and Jensen[7] considered the exit times for a class of piecewise exponential Markov processes with two-sided jumps. Kella and Stadje[8] and Perry and Stadje[9] mainly some exit times of the processes with compound Poisson process.

The remainder of the paper is organized as follows. In section 2 we give some preliminaries of the diffusion process with constant interest. In section 3 we obtain the LST of some exit times.

\section{Preliminaries}

The model (1) is a time-homogeneous Markov process (see Klebaner[10]) taking values in $\mathbb{R}$ with generator $A$ that satisfies 


$$
A f(x)=\frac{\sigma^{2}}{2} f^{\prime \prime}(x)+(c+r x) f^{\prime}(x)
$$

where $f$ belongs to the domain $D(A)$ of the generator $A$ of $\{U(t), t \geq 0\}$. Furthermore $(U(t), t)$ is also Markovian with generator $A^{\prime}$ that satisfies

$$
A^{\prime} h(x, t)=A h(x, t)+\frac{\partial}{\partial t} h(x, t) .
$$

If $h(x, \cdot)$ has a continuous first derivative for each $x$ and for each $t, h(\cdot, t)$ is in the domain of $A$, then $h(x, t) \in D\left(A^{\prime}\right)$. Denote by $F_{t}=\sigma\{U(s), 0<s \leq t\}$ the natural filtration. For later use, we give the following Lemma.

Lemma 2.1 If $h(x, t)$ is a twice continuously differentiable in $x$ and once in $t$ function with bounded first derivative in $x$, then $h(x, t) \in D\left(A^{\prime}\right)$ and furthermore

$$
M_{h}(t)=h(U(t), t)-\int_{0}^{t} A^{\prime} h(U(s), s) d s
$$

is a martingale.

In order to obtain the LST of the first exit time $T_{a, b}$, for any $\alpha>0$, we will try to find a solution to the equation

$$
A f(x)=\alpha f(x)
$$

that is

$$
\frac{\sigma^{2}}{2} f^{\prime \prime}(x)+(c+r x) f^{\prime}(x)=\alpha f(x)
$$

(3) is a second order linear differential equation, which has two positive independent solutions $f_{1}, f_{2}$ such that $f_{1}$ is strictly decreasing and $f_{2}$ is strictly increasing. Then every solution is a linear combination of the form

$$
C_{1} f_{1}(x)+C_{2} f_{2}(x)
$$

where $C_{1}, C_{2}$ are arbitrary constants. From Cai et al.[11], we know that

and

$$
f_{1}(x)=\exp \left\{-\frac{(c+r x)^{2}}{r \sigma^{2}}\right\} U\left(\frac{1}{2}+\frac{\alpha}{2 r}, \frac{1}{2}, \frac{(c+r x)^{2}}{r \sigma^{2}}\right),
$$

$$
f_{2}(x)=(c+r x) \exp \left\{-\frac{(c+r x)^{2}}{r \sigma^{2}}\right\} M\left(1+\frac{\alpha}{2 r}, \frac{3}{2}, \frac{(c+r x)^{2}}{r \sigma^{2}}\right),
$$

where $M$ and $U$ are called the confluent hypergeometric functions of the first and second kind respectively. It is easy to verify that $f_{1}(x) \rightarrow 0$ as $x \rightarrow+\infty$. More details on confluent hypergeometric functions can be found in Abramowitz and Stegun[12].

\section{The LST of some exit times}

Theorem 3.1 Given that the initial state $-\frac{c}{r}<a<u$, the LST of the time to hit $a$ is given by

$$
E_{u}\left[e^{-\alpha T_{a}}\right]=\frac{f_{1}(u)}{f_{1}(a)} .
$$

Proof. Assume that $h(x, t)$ takes the form $h(x, t)=e^{-\alpha t} f_{1}(x)$, it follows from Lemma 2.1 that $h(x, t)=e^{-\alpha t} f_{1}(x)$ is in the domain of $A^{\prime}$ and

$$
A^{\prime} h(x, t)=A h(x, t)+\frac{\partial}{\partial t} h(x, t)=0 .
$$

By Dynkin's formula, we conclude that 


$$
e^{-\alpha t} f_{1}(U(t))-f_{1}(U(0))=h(U(t), t)-h(U(0), 0)-\int_{0}^{t} A^{\prime} h(U(s), s) d s
$$

is a zero-mean martingale. Thus, for stopping time $T_{a}$ and initial condition $u$, we have that

$$
E_{u}\left[e^{-\alpha\left(t \wedge T_{a}\right)} f_{1}\left(U\left(t \wedge T_{a}\right)\right)\right]=f_{1}(u) .
$$

Because $f_{1}(x)$ is bounded on the range of possible values of $\left\{U\left(t \wedge T_{a}\right), t \geq 0\right\}$, letting $t \rightarrow+\infty$ in (5), dominated convergence theorem yields

$$
E_{u}\left[e^{-\alpha T_{a}} f_{1}\left(U\left(T_{a}\right)\right]=f_{1}(u),\right.
$$

so that

$$
E_{u}\left[e^{-\alpha T_{a}}\right]=\frac{f_{1}(u)}{f_{1}(a)}
$$

This completes the proof.

Theorem 3.2 For $b<u<a$, the LST of the first exit time from the upper barrier $a$ is given by

$$
E_{u}\left[e^{-\alpha T_{a}} 1\left(T_{a}<T_{b}\right)\right]=\frac{f_{3}(u)}{f_{3}(a)},
$$

Where $f_{3}(x)=C_{1} f_{1}(x)+C_{2} f_{2}(x)$ and $C_{1}, C_{2}$ satisfy $f_{3}(b)=C_{1} f_{1}(b)+C_{2} f_{2}(b)=0$.

Proof. It follows from Lemma 2.1 that $h(x, t)=f_{3}(x) e^{-\alpha t}$ is in the domain of $A^{\prime}$ and

$$
A^{\prime} h(x, t)=A h(x, t)+\frac{\partial}{\partial t} h(x, t)=0 .
$$

By Dynkin's formula, we conclude that

$$
f_{3}(U(t)) e^{-\alpha t}-f_{3}(U(0))=h(U(t), t)-h(U(0), 0)-\int_{0}^{t} A^{\prime} h(U(s), s) d s
$$

is a zero-mean martingale. Thus, for stopping time $T_{a, b}$ and initial condition $u$, we have that

$$
E_{u}\left[f_{3}\left(U\left(t \wedge T_{a, b}\right)\right) e^{-\alpha\left(t \wedge T_{a, b}\right)}\right]=f_{3}(u) .
$$

Because $f_{3}(x)$ is bounded on the range of possible values of $\left\{U\left(t \wedge T_{a, b}\right), t \geq 0\right\}$, letting $t \rightarrow+\infty$ in (7), dominated convergence theorem yields

$$
E_{u}\left[f_{3}\left(U\left(T_{a, b}\right)\right) e^{-\alpha T_{a, b}}\right]=f_{3}(u),
$$

hence

$$
E_{u}\left[f_{3}\left(U\left(T_{a}\right)\right) e^{-\alpha T_{a}} 1\left(T_{a}<T_{b}\right)\right]+E_{u}\left[f_{3}\left(U\left(T_{b}\right)\right) e^{-\alpha T_{b}} 1\left(T_{b}<T_{a}\right)\right]=f_{3}(u),
$$

thus

$$
E_{u}\left[f_{3}(a) e^{-\alpha T_{a}} 1\left(T_{a}<T_{b}\right)\right]=f_{3}(u),
$$

so that

$$
E_{u}\left[e^{-\alpha T_{a}} 1\left(T_{a}<T_{b}\right)\right]=\frac{f_{3}(u)}{f_{3}(a)}
$$

This completes the proof.

Using the same argument, we can obtain the following Theorem.

Theorem 3.3 For $b<u<a$, the LST of the first exit time from the lower barrier $b$ is given by

$$
E_{u}\left[e^{-\alpha T_{b}} 1\left(T_{b}<T_{a}\right)\right]=\frac{f_{4}(u)}{f_{4}(b)},
$$

Where $f_{4}(x)=C_{1} f_{1}(x)+C_{2} f_{2}(x)$ and $C_{1}, C_{2}$ satisfy $f_{4}(a)=C_{1} f_{1}(a)+C_{2} f_{2}(a)=0$. 


\section{Acknowledgements}

This work was financially supported by MOE (Ministry of Education in China) Youth Project of Humanities and Social Sciences (Project No.14YJCZH048), Hunan Provincial Natural Science Foundation of China(Grant No.13JJ5043) and Mathematics and Interdisciplinary Sciences Project, Central South University.

\section{References}

[1] Alili, L., Patie, P., Pedersen, J.L. Representations of the first hitting time density of an Ornstein-Uhlenbeck process. Stochastic Models 21, 967-980. (2005)

[2] Chiu, S.N., Yin, C.C. On occupation times for a risk process with reserve-dependence premium. Stochastic Models 18, 245-255. (2002)

[3] Chiu, S.N., Yin, C.C. The first exit time and ruin time for a risk process with reserve-dependent income. Statistics \& Probability Letters 60, 417-424. (2002)

[4] Chiu, S.N., Yin, C.C. Passage times for a spectrally negative Lévy process with applications to risk theory. Bernoulli 11(3), 511-522. (2005)

[5] dos Reis, A.D.E. How long is the surplus below zero? Insurance: Mathematics and Economics 12, 23-38. (1993)

[6] Gerber, H.U. When does the surplus reach a given target? Insurance: Mathematics and Economics 9, 115-119. (1990)

[7] Jacobsen, M., Jensen, A.T. Exit times for a class of piecewise exponential Markov processes with two-sided jumps. Stochastic Processes and their Applications 117, 1330-1356. (2007)

[8] Kella, O., Stadje, W. On hitting times for compound Poisson dams with exponential jumps and linear release rate. Journal of Applied Probability 38, 781-786. (2001)

[9] Perry, D., Stadje, W., Zacks, S. First exit times for Poisson shot noise. Stochastic Models 17, 25-37. (2001)

[10] Klebaner, F.C. Introduction to Stochastic Calculus with Applications. Imperial College Press. (1998)

[11] Cai, J., Gerber, H.U., Yang, H.L. Optimal dividends in an Ornstein-Uhlenbeck type model with credit and debit interest, North American Actuarial Journal 10, 94-119. (2006)

[12] Abramowitz, M., Stegun, I.A. Handbook of Mathematical Functions: with Formulas, Graphs, and Mathematical Tables, United States Department of Commerce, U.S. Government Printing Office, Washington, D.C.. (1972) 